\title{
VLF/ELF wave activity in the vicinity of the polar cusp: Cluster observations
}

\author{
N. Lin ${ }^{1}$, E. S. Lee ${ }^{1}$, J. McFadden ${ }^{1}$, G. Parks ${ }^{1}$, M. Wilber ${ }^{1}$, M. Maksimovic ${ }^{2}$, N. Cornilleau-Wehrlin ${ }^{3}$, A. Fazakarley ${ }^{4}$, \\ E. Lucek ${ }^{5}$, H. Rème ${ }^{6}$, O. Santolik ${ }^{7}$, and Q.-G. Zong ${ }^{8,}{ }^{*}$ \\ ${ }^{1}$ Space Sciences Laboratory, UC Berkeley, Berkeley, CA 94720, USA \\ ${ }^{2}$ LESIA, Observatoire de Paris, 92195 Meudon Cedex, France \\ ${ }^{3}$ CETP/UVSQ, 10-12 Av. De l'Europe, 78140 Velizy, France \\ ${ }^{4}$ Mullard Space Science Laboratory, Univ. College, London, UK \\ ${ }^{5}$ Space and Atmospheric Physics, Imperial College, London, SW7 2BZ, UK \\ ${ }^{6}$ CESR, 9 ave du Colonel Roche, 31028Toulouse Cedex 4, Toulouse, France \\ ${ }^{7}$ Faculty of Mathematics and Physics, Charles University, Prague, Czech Republic \\ ${ }^{8}$ Center for Space Physics, Boston University, Boston, MA 02215, USA \\ *now at: Center for Atmospheric Research, University of Massachusetts Lowell, Lowell, MA 01854, USA
}

Received: 6 January 2006 - Revised: 16 May 2006 - Accepted: 6 June 2006 - Published: 9 August 2006

\begin{abstract}
Observations by the Cluster spacecraft of VLF/ELF wave activity show distinct signatures for different regions in the vicinity of high altitude polar cusps, which are identified by using magnetic field and plasma data along spacecraft trajectories. These waves include: (1) Broad band magnetic noise observed in the polar cusp at frequencies from several $\mathrm{Hz}$ to $\sim 100 \mathrm{~Hz}$, below the local electron cyclotron frequency, $f_{c e}$. Similar magnetic noise is also observed in the high latitude magnetosheath and the magnetopause boundary layer. (2) Strong broad band electrostatic emissions observed in the cusp, in the magnetosheath, and in the high latitude magnetopause boundary layer, at frequencies extending from several $\mathrm{Hz}$ to tens of $\mathrm{kHz}$, with maximum intensities below $\sim 100 \mathrm{~Hz}$. (3) Narrow-band electromagnetic whistler waves at frequencies $\sim 0.2-0.6 f_{c e}$, frequently observed in the closed boundary layer (CBL) adjacent to the polar cusp. These waves are for the first time observed in this region to be accompanied by counter-streaming electron beams of $\sim 100 \mathrm{eV}$, which suggests that the waves are excited by these electrons through wave-particle interaction. (4) Narrow-band electrostatic waves observed slightly above the local $f_{c e}$ in the CBL. (5) Lion roars, observed in the high latitude magnetosheath, often in magnetic troughs of mirror mode oscillations. The above wave signatures can serve as indicators of the regions in the vicinity of the magnetospheric cusp.
\end{abstract}

Keywords. Magnetospheric physics (Magnetopause, cusp, and boundary layers; Plasma waves and instabilities) - Space plasma physics (Wave particles interactions)

Correspondence to: N. Lin

(nlin@ssl.berkeley.edu)

\section{Introduction}

It has been known that the magnetospheric cusp and its vicinity are rich in VLF/ELF wave activity with frequencies ranging from $\sim 100 \mathrm{~Hz}$ to several tens $\mathrm{kHz}$ (cf. Scarf et al., 1972, 1974; Gurnett and Frank, 1978; Pottelette et al., 1990; Lakhina et al., 2000; Tsurutani et al., 2001; Pickett et al., 2001, 2002; Menietti et al., 2002a, b). These waves are closely involved in the interaction with charged particles in the cusp regions. The generation mechanisms of these waves are not all understood and need more investigation. The polar orbit of the Cluster spacecraft has provided excellent opportunities to study the wave activity in the cusp regions. In this paper we will present a study of two high altitude cusp crossings.

As will be shown below, during the two crossings, the spacecraft have traversed various regions including the plasma mantle, the cusp proper, dayside magnetospheric boundary layers at high latitudes, the magnetopause and the magnetosheath. In general, not all the regions which have been clearly identified in the two events can be observed in a polar crossing. Depending on the spacecraft trajectory, the tilt of the Earth's dipole, and geomagnetic activity, a spacecraft's polar crossing may miss some of the regions. Thus, the observation of waves occurring along the spacecraft trajectories in our study may provide more comprehensive reference for cusp waves than previous studies.

In this paper, we will first identify various regions in the vicinity of the cusp along the spacecraft trajectory by using the observations of magnetic field and plasma signatures, then display the waves observed within these regions. Among these waves, we analyze in details the whistler waves

Published by Copernicus GmbH on behalf of the European Geosciences Union. 


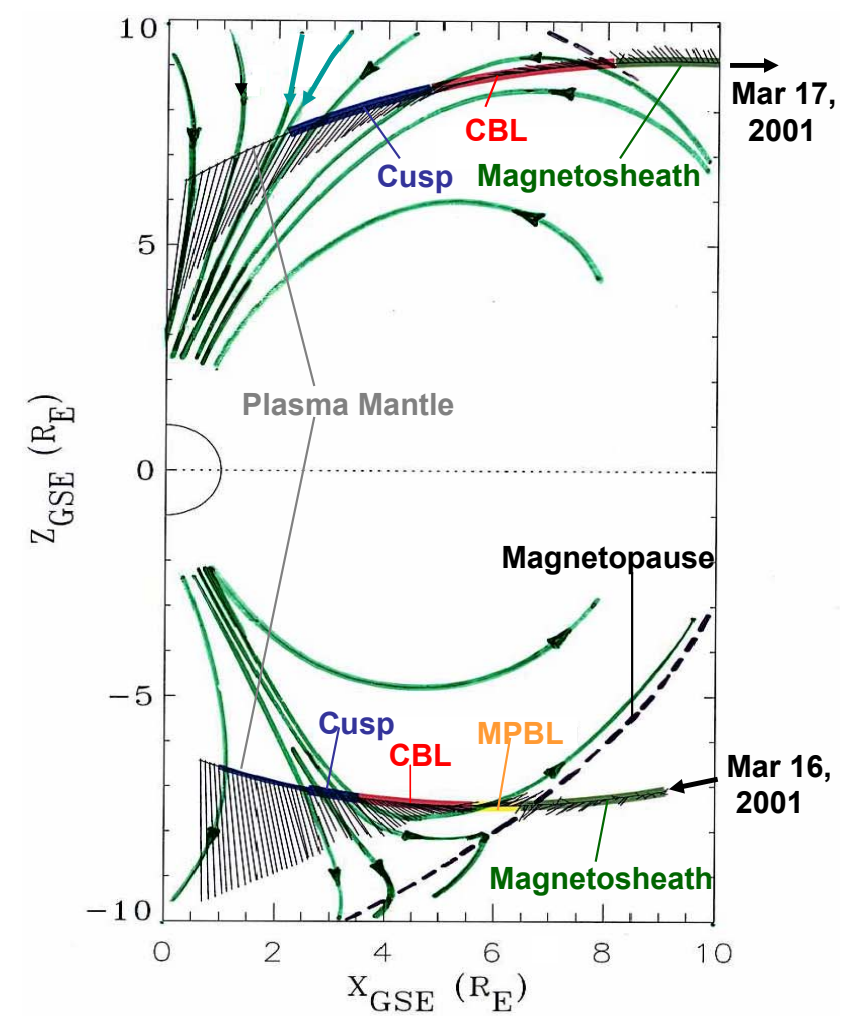

Fig. 1. Trajectories of the Cluster spacecraft for 16 March 2001, 16:00-21:00 UT, and for 17 March 2001, 05:00-10:00 UT, projected onto the $\mathrm{x}-\mathrm{z}$ plane in GSE coordinates. Measured magnetic field vectors in the $\mathrm{x}-\mathrm{z}$ plane are overplotted along the trajectories. Also overplotted is a cartoon of magnetic field lines which are consistent with the direction of the measured field vectors on the trajectories. The dashed lines represent the magnetopause. Different colors mark various regions, which include the magnetosheath, closed boundary layer (CBL), the cusp, Magnetopause boundary layer (MPBL), and the plasma mantle.

occurring in the closed boundary layer, which are coincident with the existence of counter-streaming electron beams of $\sim 100 \mathrm{eV}$. It is the first simultaneous observations of whistler waves and electron beams in the high latitude closed boundary layer, which indicate a wave particle interaction occurring in that region. In the Summary section, we will compare our observations with previous studies and discuss mechanisms generating some of the waves.

The wave data we used are obtained from Cluster's Spatio Temporal Analysis of Field Fluctuations and Spectrum Analyzer (STAFF-SA) experiment (Cornilleau-Wehrlin et al., 1997, 2003). The STAFF spectrum analyzer measures the $5 \times 5$ components of the spectral matrix formed by the three STAFF wave magnetic components and the two spin wave electric components from the EFW experiment (Gustafsson et al., 2001), in the frequency range of $8 \mathrm{~Hz}$ to $4 \mathrm{kHz}$ in 27 channels. The ion data, which include the ion energy flux, the plasma density, velocity and temperature, were ob- tained by the Cluster Ion Spectrometry (CIS) experiment (Réme et al., 1997, 2001). The electron measurements are from the Plasma Electron and Current Experiment (PEACE) measurements (Johnstone et al., 1997), and the DC magnetic field data are from The Cluster Magnetic Field Investigation (FGM) (Balogh et al., 1997).

\section{Observations and analysis}

The two intervals to be studied are the polar crossings on 16 March 2001, 16:00-21:00 UT, and on 17 March 2001, 05:00-10:00 UT. Figure 1 shows the trajectories of the Cluster spacecraft during these two intervals, projected onto the $\mathrm{x}-\mathrm{z}$ plane in GSE coordinates. Measured magnetic field vectors in the $\mathrm{x}-\mathrm{z}$ plane are overplotted along the trajectories every $3 \mathrm{~min}$ for the 16 March event and every $5 \mathrm{~min}$ for the 17 March event. A cartoon of magnetic field lines which are consistent with the direction of the measured field vectors on the trajectories are also overplotted to illustrate the magnetic field configuration during the events. Various regions identified according to field and plasma signatures are marked with different colors. In between the cusp and the magnetosheath are the magnetopause boundary layer (MPBL) and the closed boundary layer (CBL). These regions will be defined in the context of Cluster observations in the sections immediately following. A study by Onsager et al. (2001) used particle observations from Polar to make similar identifications of cuspregion boundary layers.

\subsection{Cusp crossing on 17 March 2001, 05:00-10:00 UT}

During this period Cluster was traveling outbound in the northern hemisphere crossing the cusp at about 8 to $9 R_{E}$ from the Earth. After shifting in time to account for propagation from the observation point to Earth, the interplanetary magnetic field (IMF) measured by the ACE spacecraft for this period (not shown) was mostly northward, except for a brief $(\sim 10 \mathrm{~min})$ southward turning at $\sim 05: 30$ UT and at $\sim 06: 40$ UT (for about $5 \mathrm{~min}$ ). The observations of the cusp in this event from the particle point of view, and its relation with the IMF, has been studied in depth by Vontrat-Reberac et al. (2003).

\subsubsection{Identifying various regions near the cusp}

Figure 2 shows the observations of ion energy flux, density, velocity, temperature, and magnetic field variations during the period. Coming out from the plasma mantle, the spacecraft entered the cusp rather abruptly at $\sim 05: 07$ UT. The cusp was characterized by magnetosheath-like plasma, with the density $\left(\sim 10-20 / \mathrm{cm}^{3}\right)$ comparable to that of the magnetosheath, and the peak energy flux mainly resulting from ions of a few hundred $\mathrm{eV}$, also similar to that of the magnetosheath (the interval after 09:21 UT). At the cusp entry the plasma was flowing parallel to the magnetic field with a velocity 


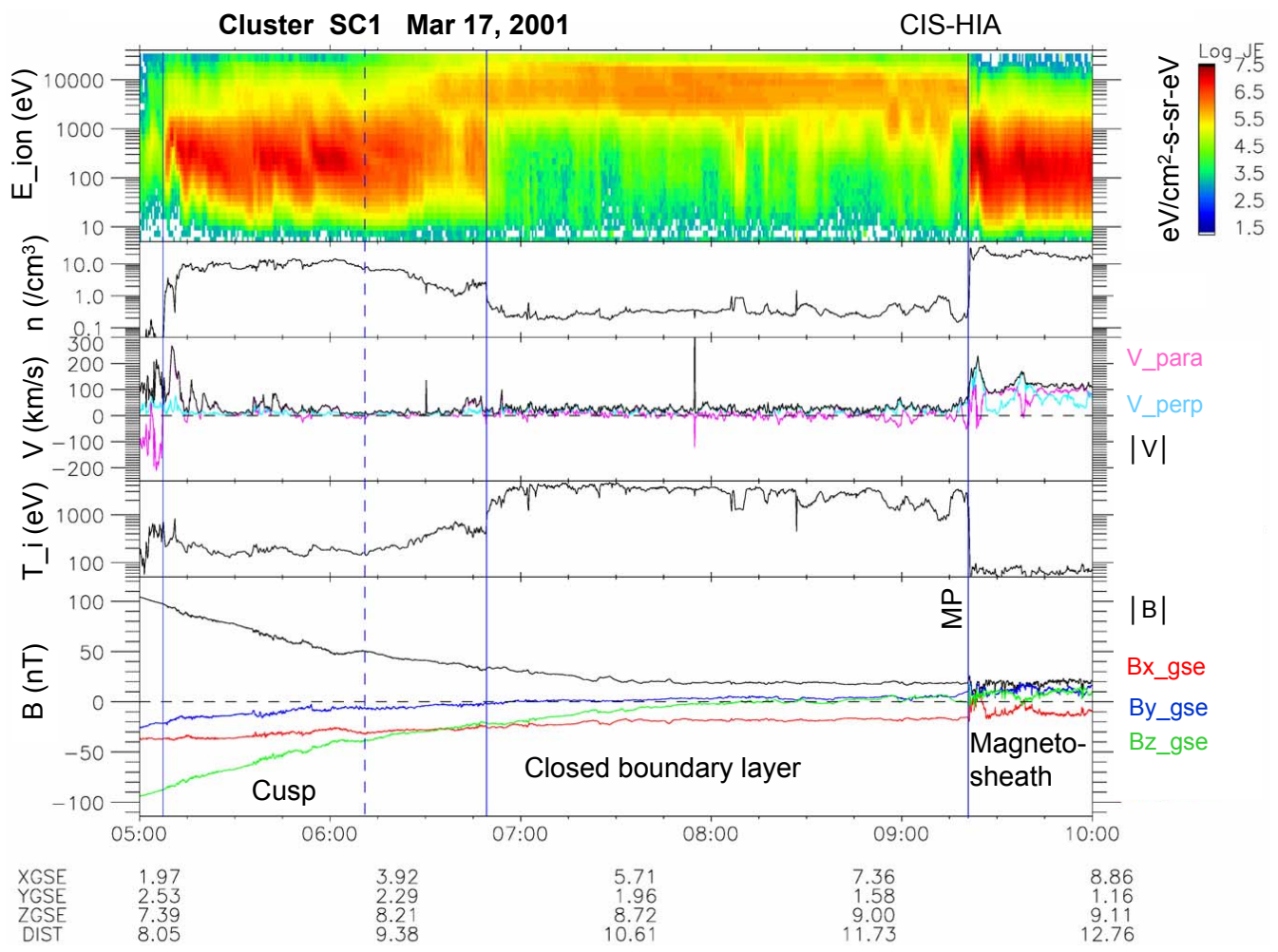

Fig. 2. From top to bottom: The ion energy flux, density, velocity (plotted as components parallel, in red, and perpendicular, in blue, to the ambient magnetic field, and the total velocity in black), temperature, and the magnetic field (in GSE coordinates) variations during the period of 17 March 2001, 05:00-10:00 UT. Vertical lines mark different regions traversed by the spacecraft.

up to $\sim 200 \mathrm{~km} / \mathrm{s}$, and then a smaller flow velocity was observed. The cusp was also characterized by stagnant plasma flow with almost zero ion velocity in most of the indicated interval, similar to the stagnant region reported by Lavraud et al. (2004), while in the magnetosheath, the plasma flowed with a significant component parallel to the magnetic field lines, with some variations, at $100-200 \mathrm{~km} / \mathrm{s}$. The magnetic field showed enhanced fluctuations in the cusp interval (0507 to $\sim 06: 50 \mathrm{UT}$ ).

At $\sim 06: 50$ UT, Cluster entered a new region which had significant energetic ions of up to $\sim 30 \mathrm{keV}$ as seen from the first panel of Fig. 2. This is a region consisting of mainly closed field lines, since PEACE detected trapped and counter-streaming electrons of keV energy (not shown). The enhancement of energetic ( $>20 \mathrm{keV}$ ) electron flux in this region (Zong et al., 2005) also suggests that it is a region containing a magnetospheric population. This region could be the outer part of the dayside plasma sheet (see, for example, Fig. 5.1 of Potemra, 1994). Since this is a region close to the cusp and high latitude magnetosheath (see Fig. 1), and the ion population shows a mix of hot magnetospheric ions and magnetosheath-like ions of a few hundreds $\mathrm{eV}$, we will identify this region as the closed boundary layer (CBL), a name given to the dayside high latitude boundary layer between the magnetosphere and the cusp (Crooker et al., 1977; see also
Fig. 1 of Tsurutani et al., 2001). This region is similar to the outer boundary of the "region 4" identified in Onsager et al. (2001). The motion of the boundary led to frequent spacecraft approaches to the adjacent cusp and magnetosheath regions for brief periods, resulting in enhancements of hundred $\mathrm{eV}$ ion fluxes, which are seen most clearly between 08:00 and 09:21 UT. The plasma velocity in this region was near zero. The enhancements in $100 \mathrm{eV}$ ion fluxes were accompanied by density increases and temperature decreases, both consistent with mixing of sheath or cusp plasma, and another indication of boundary layer behavior. In CBL the DC magnetic field had less and weaker fluctuations, compared to the magnetic filed in the cusp and magnetosheath. Between 06:10 UT (the vertical dashed line) and 06:50 UT, the spacecraft seemed to be in a transition region between the cusp and the closed boundary layer, where the magnetosheath-like ions and day-side plasma sheet ions are mixed, with magnetosheath particle fluxes decreasing and dayside plasmasheet particle flux increasing, and the ion density and temperature gradually change from the cusp level to CBL level.

At 09:21 UT a sudden boundary motion resulted in the spacecraft crossing into the magnetosheath, where the magnetic field became more turbulent with intense compressional fluctuations, as seen from the variations of magnetic field magnitude in the last panel of Fig. 2. 


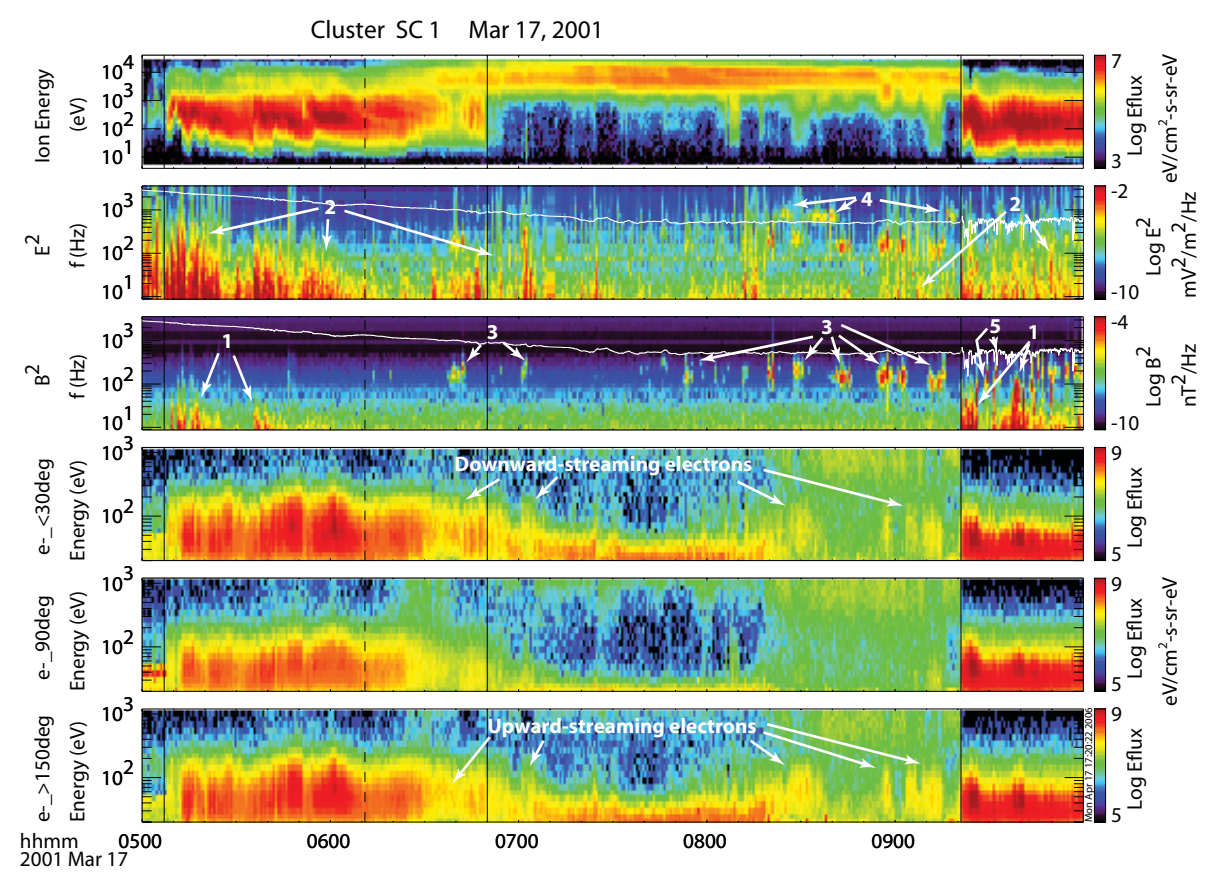

Fig. 3. From top to bottom: (1) The ion energy flux (the same as that in Fig. 2); (2) The sum of the electric field power spectral density (in $\mathrm{mV}^{2} / \mathrm{m}^{2} / \mathrm{Hz}$ ); (3) The sum of the magnetic field power spectral density (in $\mathrm{nT}^{2} / \mathrm{Hz}$ ); (4)-(6) Energy spectra of electrons for pitch angle $\alpha<30^{\circ}, 70^{\circ}<\alpha<110^{\circ}$, and $\alpha>150^{\circ}$, respectively. The vertical black lines marked the boundaries between different regions as in Fig. 1. Numbers in panels 2 and 3 mark wave mode observed: "1" for the broadband magnetic noise; " 2 " for the broadband electrostatic emissions; " 3 " for the CBL whistler mode waves; " 4 " for the narrow-band electrostatic whistler waves; and "5" for the lion roars.

\subsubsection{VLF/ELF wave activity}

The wave activity during this period is displayed in panels 2 and 3 of Fig. 3. The electric field power spectral density (in $\mathrm{mV}^{2} / \mathrm{m}^{2} / \mathrm{Hz}$ ) shown in panel 2 is the sum of the measurements in the spin plane, which is roughly in the GSE $x-y$ plane, and panel 3 shows the sum of the magnetic wave power spectral density (in $\mathrm{nT}^{2} / \mathrm{Hz}$ ) of three components. The vertical black lines mark the boundaries between different regions as in Fig. 2.

Distinct wave modes are observed in the various regions along the trajectory. In the cusp region, frequent occurrences of sporadic bursts of broad-band magnetic noise at frequencies ranging from the lower limit of the instruments ( $8 \mathrm{~Hz}$ ) to $\sim 100 \mathrm{~Hz}$ are observed (marked with number " 1 " in panel 3). Similar magnetic waves also occur in the magnetosheath. The frequencies of these magnetic noises are always below the local $f_{c e}$, which is overplotted as the white curves in panels 2 and 3. We notice a magnetic depression region in the magnetosheath just outside of the magnetopause, as seen in panel 3 of Fig. 3 as a dip in the $f_{c e}$ line, or in the last panel of Fig. 2 as a dip in the magnetic field strength, concurrent with the broadband magnetic waves. The mechanism of these waves which are associated with the magnetic depression will be discussed in the third section.
We also notice that short bursts of magnetic waves at $\sim 100-200 \mathrm{~Hz}$ in the cusp region (for example, near 05:28 and at $\sim 05: 46$ UT) are observed to have much weaker intensity than the broadband noise bursts. Pickett et al. (2001, 2002) reported frequent observations of electromagnetic whistler mode wave bursts obtained using high time resolution Polar plasma wave instrument (PWI) measurements that occurred on millisecond timescales around a few tens of $\mathrm{Hz}$, a few hundreds of $\mathrm{Hz}$, and just below fce in the cusp turbulent boundary layer (TBL). Field and plasma conditions of the cusp region in our event might not be the same as those of the cusp TBL region, but the weak short magnetic wave bursts seen in our event are likely to be similar to those observed at a few hundred $\mathrm{Hz}$ by Pickett et al. $(2001,2002)$ In our event, these short bursts of whistler waves are not well resolved in the STAFF spectrograms, probably because they are detected at the same time and in the same frequency range as the broadband magnetic noise and their intensities are lower than those of the broadband noise. In this study we will not discuss further these short burst whistler waves. Pickett et al. (2001, 2002) suggested that these waves are generated at reconnection sites and propagated away from the sites along reconnection field files.

In the cusp and the magnetosheath regions, we also observed broad-band electrostatic waves covering the entire frequency range of the STAFF instrument (marked with num- 
ber "2" in the second panel). The peak power observed was at a few hundred $\mathrm{Hz}$ and below, but declining wave activity extended at least to the upper limits of the detector's range. Observations from Cluster's Whisper instrument (not shown here), which measures electric wave signals from 2$80 \mathrm{kHz}$, showed that the electrostatic waves were observed up to $20-30 \mathrm{kHz}$. Enhancements of these electrostatic waves were also observed in the transition regions between the cusp (or the magnetosheath) and the CBL, which are characterized by the coexistence of magnetospheric $\mathrm{keV}$ ions and a few hundred $\mathrm{eV}$ of magnetosheath ions (panel 1). In the CBL their intensity was much reduced.

Observations from the Polar satellite (e.g. Franz et al., 1998; Tsurutani et al., 1998a; Lakhina et al., 2004; Cattell et al., 1999) have revealed that electric field structures or solitary waves moving along magnetic field lines in the cusp regions may cause observed broadband electrostatic noise (BEN). These structures are usually identified as a collection of individual bipolar electric field variations in the parallel component. The durations of the pulses are $\sim 1 \mathrm{~ms}$. The electric field measurements of Cluster are made in the spin plane only. For the cusp crossing periods, it is difficult to resolve the measured electric field into parallel and perpendicular components. Although it is likely that a significant source of the broadband noise results from FFT rendering of these frequently-observed solitary waves, high-resolution waveform data from Cluster/Wideband were not available for the intervals we examined, so we cannot confirm their presence.

In the magnetosheath (after 09:21 UT), we also saw short bursts of narrow-banded magnetic waves at a few hundred $\mathrm{Hz}$ (marked with number " 5 " in panel 3), often occurring at the dips of the ambient magnetic field and thus in the dips in $f_{c e}$ line in panel 3. These are the "lion roar" whistler mode waves which are commonly seen in the low latitude magnetosheath, and are often coupling with mirror mode waves (cf. Tsurutani et al., 1982; Zhang et al., 1998; Maksimovic et al., 2001; Yearby et al., 2005).

A striking feature of wave activity in the CBL was the occurrence of narrow-band electromagnetic whistler waves, which can be seen below $f_{c e}$ at a few hundred $\mathrm{Hz}$ (marked with number " 3 " in panel 3). These waves were seen in both magnetic and electric field components, and spread over the CBL, most clearly seen when the spacecraft seemed to be in the outer CBL, where enhancements of sheath-like ions were observed in addition to the $\mathrm{keV}$ ions (for examples, in the interval between 08:30 and 09:15 UT).

It is interesting to find that the occurrences of the above CBL whistler waves were accompanied by the existence of counter-streaming electrons of $\sim 100 \mathrm{eV}$. The last three panels of Fig. 3 show the spectra of electron energy fluxes detected by the LEEA sensor-head of the PEACE instrument, which cover the lower part of the energy range in 30 channels $(\sim 3 \mathrm{eV}$ to $1045 \mathrm{eV})$. Panels 4,5 , and 6 display the energy flux of electrons with pitch angle $\alpha<30^{\circ}, 70^{\circ}<\alpha<110^{\circ}$, and $\alpha>150^{\circ}$, respectively. Only the energy fluxes of electrons above $20 \mathrm{eV}$ are shown, because there is significant contamination from photoelectrons in lower than $20 \mathrm{eV}$ data.

The electron data show that in the intervals when we saw the bursts of CBL whistler waves, there were enhancements in parallel and anti-parallel electron energy fluxes (panels 4 and 6), but not in trapped electrons (panel 5). This is most clearly seen for the wave bursts between 08:00 and 09:20 UT, and that at about 06:40 UT in the transition region. The energy of the counter-streaming electrons which are associated with the waves ranges from several tens $\mathrm{eV}$ to $\sim 200 \mathrm{eV}$. In this event, the anti-parallel fluxes seemed to be more intense than the parallel fluxes. Since the spacecraft was in the northern hemisphere, the anti-parallel electron fluxes were originated from the northern ionosphere, while the parallel fluxes may have been from the electrons reflected from the conjugate ionosphere. We show in Sect. 3 that these waves can be identified as a whistler mode generated by a resonant interaction with the electron beams observed simultaneously.

There is another wave mode seen in the CBL, narrowband electrostatic waves at frequencies slightly above local $f_{c e}$. These waves are most clearly seen in electric field spectra (marked with number " 4 " in the second panel) between 08:20 and 08:45 UT and at $~ 09: 18$ UT, and no corresponding magnetic field component observed.

\subsection{Cusp crossing on 16 March 2001, 16:00-21:00 UT}

During this period Cluster was travelling inbound in the southern hemisphere crossing the cusp at about 9 to $8 R_{E}$ from the Earth. The interplanetary magnetic field (IMF) measured by ACE spacecraft for this period (not shown) was mostly southward except for intervals between 17:30 and 17:45 UT, between 18:00 and 19:00 UT, and a few other short period of a few minutes, when the IMF turned northward.

\subsubsection{Various regions in the cusp vicinity}

Figure 4 show observations of the ion plasma and the magnetic field for the period in the same format as in Fig. 2. Travelling in the magnetosheath (before $\sim 17: 08 \mathrm{UT}$ ), the spacecraft observed ion plasma dominated by a few hundred $\mathrm{eV}$ ions, with background ion velocity of $100-200 \mathrm{~km} / \mathrm{s}$, typical for magnetosheath plasma flows. On top of the magnetosheath flow, there were ions frequently observed to be streaming in an anti-parallel direction, as seen as spikes in ion velocity in panel 3 . These streaming ions had much higher velocity than the magnetosheath flow, suggesting that there were source regions north of the spacecraft which accelerate the ions. Considering the IMF being southward during this period, it is likely that reconnection processes at the magnetopause were generating these ion bursts. These ion bursts may intensify wave activity in this region.

At $\sim 17: 08$ UT, the spacecraft crossed the magnetopause. Unlike in the first event, where the spacecraft crossed the 


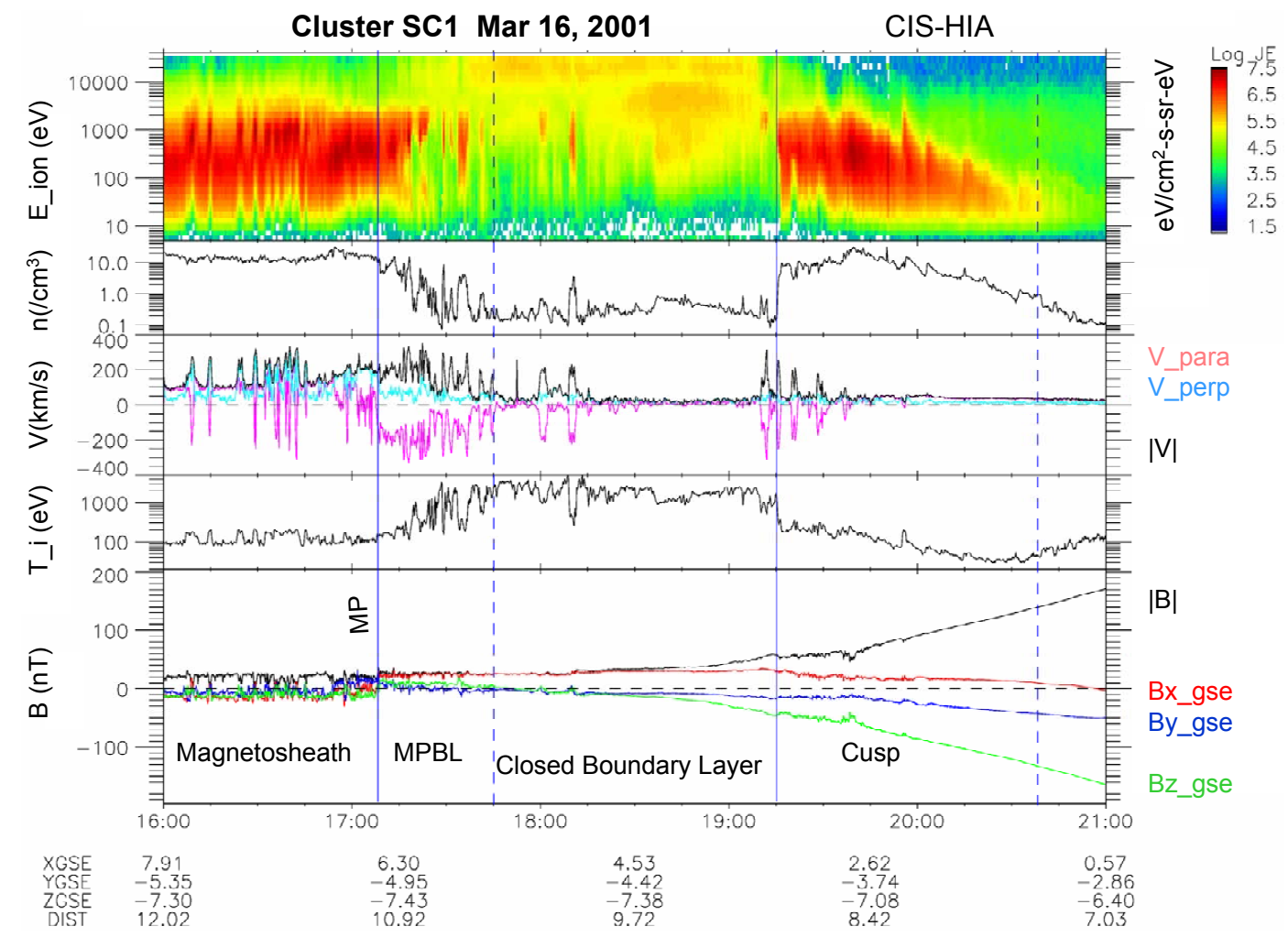

Fig. 4. The same as Fig. 2, but for the period of 16 March 2001, 16:00-21:00 UT.

magnetopause abruptly without seeing a significant boundary layer, in this event the spacecraft entered a magnetopause boundary layer (MPBL) after crossing the magnetopause. The MPBL was characterized by a mix population of magnetosheath ions and more energetic (a few tens $\mathrm{keV}$ ) magnetospheric ions (see panel 1). The density and ion temperature also gradually changed, with fluctuations, from the magnetosheath levels to that of the adjacent closed boundary layer. We notice that in the MPBL bursts of accelerated ions, which are probably of the same nature as those velocity spikes seen in the magnetosheath period, are more frequently observed, suggesting that the reconnection process may have been very active during the period.

At $\sim 17: 45$ UT the spacecraft entered the CBL and traveled in the region until $\sim 19: 15$ UT when it entered the cusp. During this period, the spacecraft briefly entered the magnetosheath or the cusp at $\sim 18: 00,18: 10$, and 19:12 UT due to the motion of the CBL, and we saw bursts of ion streaming in these intervals similar to those seen in the MPBL. Signatures of CBL were obvious for this interval: a mixed ion population, almost zero ion velocity, low plasma density $(\sim 0.1-$ $0.5 \mathrm{~cm}^{-3}$ ), high ion temperature (several $\mathrm{keV}$ ), and a relatively quiet DC magnetic field. The RAPID data (not shown) also revealed enhanced fluxes of energetic ions and electrons in the CBL as in the case of the first event (Zong et al., 2005).
The spacecraft entered the cusp at $\sim 19: 15$, as indicated by stagnant magnetosheath-like ions. The magnetic field showed a slight depression and was more turbulent, which are typical cusp signatures. We note that several bursts of streaming ions were observed in the cusp flowing antiparallel to the ambient magnetic field, which were likely generated by reconnection process as was the case in the MPBL. As the spacecraft went further inward, from 19:45 UT the density and temperature gradually decreased, while the magnetic field increased. Unlike in the previous event, there is no clear boundary between the cusp and the plasma mantle in this southward IMF event, although the interval near 20:40 UT (marked by a vertical dashed line) appears to match such a transition.

\subsubsection{VLF/ELF wave activity}

Wave activity for the second event is displayed in Fig. 5 in the same format as that of Fig. 3. Panel 3 shows that broadband magnetic noise up to a few hundred $\mathrm{Hz}$ occurred in the magnetosheath, MPBL, and in the cusp. A period of magnetic field depression is also observed just outside the magnetopause, concurrent with the broadband magnetic waves. Broadband electrostatic waves were also observed in these three regions, including in the brief excursion of the spacecraft into these regions from the CBL, with their frequencies extended up to $\sim 20-30 \mathrm{kHz}$ (as seen in Whisper data) but 


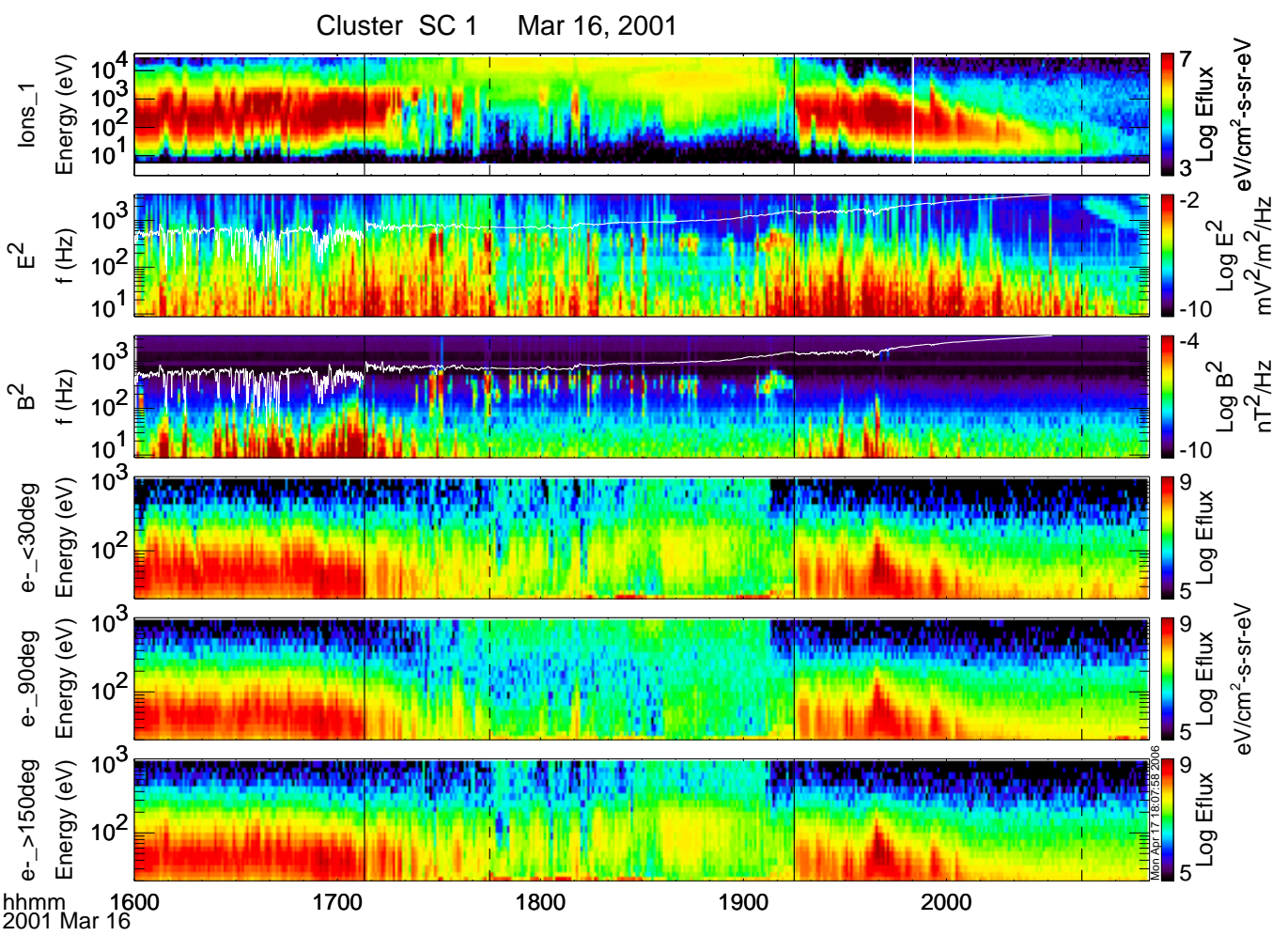

Fig. 5. The same as Fig. 3, but for the period of 16 March 2001, 16:00-21:00 UT.

peak intensities below $\sim 100 \mathrm{~Hz}$. These electrostatic waves gradually died away as the spacecraft went into the plasma mantle.

The narrow-band electromagnetic whistler waves occurring in the CBL were clearly seen at frequencies between 100 and $400 \mathrm{~Hz}$, below the local $f_{c e}$ line. They spread over the entire CBL and extended to the inner part of MPBL. As in the case of the first event, the occurrence of these CBL whistler waves were accompanied by counterstreaming electron beams (Panels 4, 5, 6 in Fig. 5).

Electrostatic waves with frequencies slightly above $f_{c e}$ have also occurred in CBL, most clearly seen at $\sim 18: 35$ and 18:20 UT. Narrow band lion roars were also seen in the magnetosheath in this event, but were much rarer compared to the first event. In the magnetosheath, dips of the ambient magnetic field are frequently observed, but in this event the dips were mostly coincident with the ion velocity spikes (see Fig. 4). The ion energy spectra show that these magnetic field dips occurred when the spacecraft went into or approached closer to the MPBL due to the motion of the magnetopause, and not the dips of mirror mode waves. That is why we did not see lion roars in most of the dips here, unlike in the first event.

\section{Summary and discussion}

We have observed a variety of VLF/ELF waves in the high altitude magnetospheric cusp and its vicinity along Cluster trajectories. In the following we summarize our observations and compare the observations with previous studies.

Broadband magnetic noise: We observe this spanning a few $\mathrm{Hz}$ to several hundred $\mathrm{Hz}$ in the cusp and in the high latitude magnetosheath near the cusp. Their frequencies are always lower than the local $f_{c e}$. They are most likely to be whistler mode waves. These waves are similar to "ULF-ELF magnetic noise" of the cusp in Hawkeye observations (Gurnett and Frank, 1978). In that study the authors suggested that the waves were uniquely associated with the cusp. In our observations we also see similar noise in the magnetosheath where plasma flows are large. From the plasma data we notice that the cusp plasma is very similar to that of magnetosheath plasma, except that the cusp plasma is stagnant. The two regions are likely connected to each other (see Fig. 1), and it is not surprising that we see similar waves in the two regions. Alfvenic turbulence (Grison et al., 2005) has been suggested as a possible energy sources for the waves.

Broadband magnetic noise from a few $\mathrm{Hz}$ up to $f_{c e}$ has also been observed almost continuously in the cusp TBL region (Pickett et al., 2001). The authors suggested that these waves are composed of whistler and kinetic Alfven 

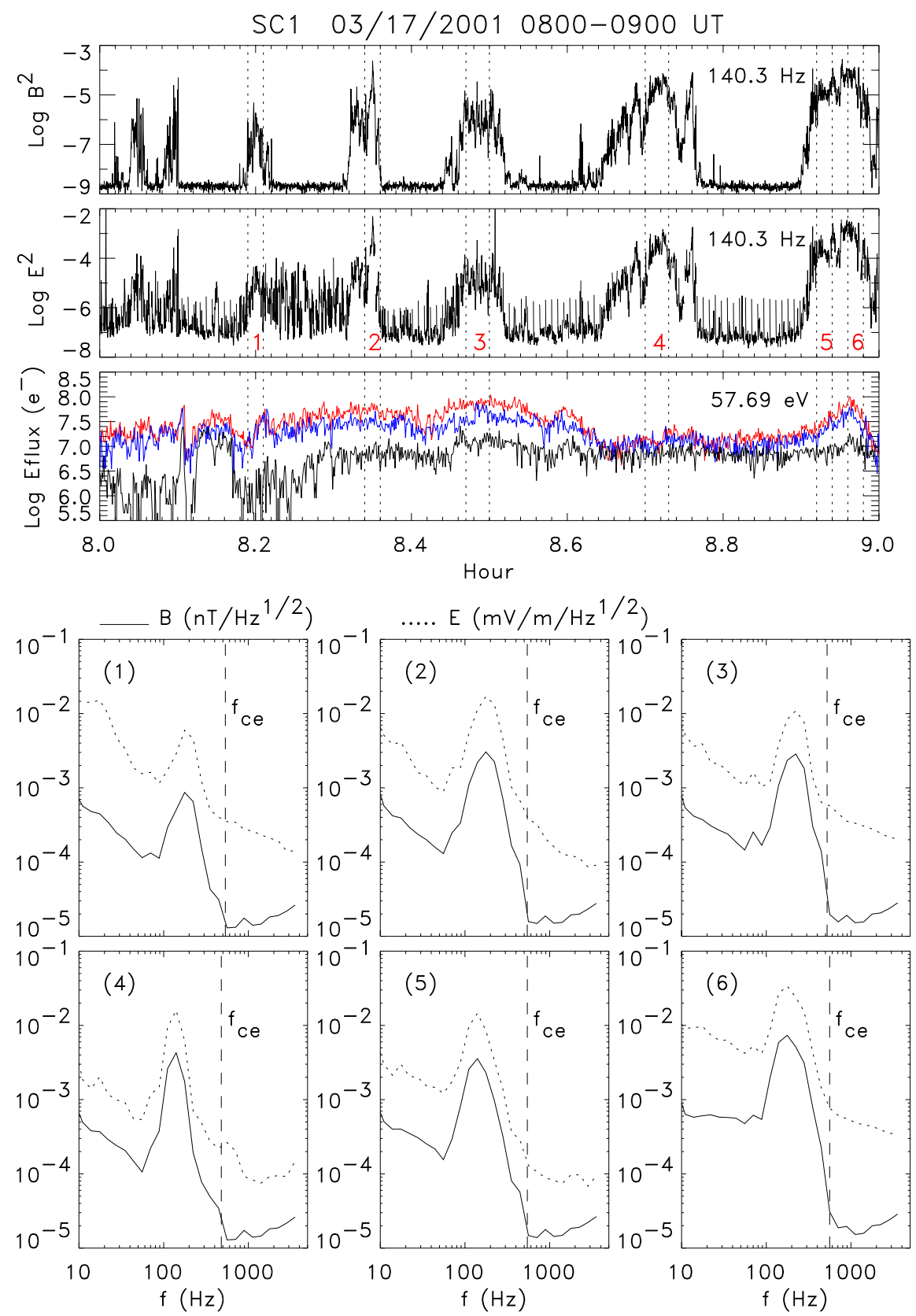

Fig. 6. Panels 1 and 2: The variations of magnetic and electric field wave power density for the period of 17 March 2001, 08:00-0900 UT, detected in a channel with central frequency of $140.3 \mathrm{~Hz}$. Panel 3: The energy fluxes of electrons in the channel of $57.69 \mathrm{eV}$ for three pitch angle ranges: $\alpha<30^{\circ}$ (blue), $70^{\circ}<\alpha<110^{\circ}$ (black), and $\alpha>150^{\circ}$ (red), respectively. Six intervals (labeled 1-6 between each pair of vertical dashed lines) are selected for detailed study. The lower part of the figure shows wave amplitude density spectra for the six intervals with the solid curves represent magnetic wave spectra and the dashed curves for the electric wave spectra. The vertical dashed line in each spectra marks the $f_{c e}$.

modes generated in the out flow region of the reconnection site closest to the spacecraft. In addition, broadband electromagnetic waves in a frequency range of $0.1-30 \mathrm{~Hz}$ have been observed associated with strong magnetic depressions in the vicinity of high-latitude magnetopause boundary (Stasiewicz et al., 2001). These waves are interpreted to be the spatial turbulence of kinetic Alfvén waves, Doppler-shifted to higher frequencies in the satellite frame by convective plasma flows. In our events, the broadband magnetic noise below $\sim 100 \mathrm{~Hz}$ in the magnetosheath near the magnetopause is likewise observed simultaneously with magnetic depressions. Tsurutani et al. (1998b) reported intense broadband 
electromagnetic plasma waves frequently observed in the polar cap boundary layer, at the location below the cusp fields. This area might correspond to the region near the boundary between the cusp and the closed boundary layer in our study. Tsurutani et al. suggested that the waves are likely a mixture of obliquely propagating electromagnetic whistler mode waves and electrostatic waves locally generated by field-aligned currents or current gradients.

Broadband electrostatic emissions at frequencies ranging from several $\mathrm{Hz}$ to about $20-30 \mathrm{kHz}$, with maximum intensity at frequencies below $100 \mathrm{~Hz}$, occur in the cusp and in the magnetosheath. Previous studies (Gurnett and Frank, 1978) have reported that the frequencies of these electrostatic emissions could reach $30-100 \mathrm{kHz}$, and a possible generation mechanism is a current driven electrostatic instability. As mentioned above, recent observations have shown that the broadband electrostatic noise in the cusp region is likely to be caused by solitary wave structures, or "electron holes", traveling along the ambient magnetic field (e.g. Franz et al., 1998; Tsurutani et al., 1998a, 2001, 2003; Pickett et al., 2001; Lakhina et al., 2000, 2004, and references therein). Similar structures have also been observed in other regions of the magnetosphere, for example, in the plasma sheet boundary layer (Matsumoto et al., 1994; Cattell et al., 1999). Lakhina et al. (2000) found that the electrostatic solitary waves (ESW) occurring in the plasmasheet boundary layer mainly contribute to the high-frequency portion of the BEN spectra, while the low-frequency part results from some other, yet-to-be-identified mode(s).

Recently, Khotyaintsev et al. (2004) reported observations of broadband electric wave emissions at frequencies extending from the electron cyclotron frequency up to the plasma frequency in the cusp/exterior-cusp region. The spectral peaks of these waves are at the plasma frequency or at frequencies below the plasma frequency. These waves seem to be different from the broadband electrostatic emissions in our events and from those observed by Gurnett and Frank (1978), whose maximum intensity occurs at much lower frequencies. Khotyaintsev et al. (2004) suggested that the high frequency waves they observed are generated by electron beams propagating along the separatrices of the reconnection region via bump-on-tail or electron two-stream instability. Two types of broadband electrostatic waves have been observed at low altitude ( $\sim 1$ to $2 R_{E}$ ) cusp/cleft regions (Pottelette, 1990). Those observed above the lower hybrid frequency, $f_{L H}$, are attributed to the lower hybrid instability generated by density gradients. The other type of the broadband electrostatic noise is observed between $f_{c e}$ and the electron plasma frequency, and is attributed to the excitation of an electron acoustic mode. In our events the broadband electrostatic emissions cover frequencies well below $f_{c e}$ and $f_{L H}$. It could be that the broadband waves are a superposition of several wave modes.
Narrow band electromagnetic whistler waves have been observed frequently in the closed boundary layer. Their frequencies are about $0.2-0.6$ of $f_{c e}$. They may also occur in the high latitude magnetopause boundary layer. Counterstreaming electron beams of $\sim 100 \mathrm{eV}$ are seen accompanying the occurrence of these CBL whistler waves. To our knowledge, these are the first observations of narrow band electromagnetic whistler waves associated with the electron beams in the high latitude CBL near the cusp and in the dayside magnetopause-cusp boundary layer. The concurrence of the waves and the electron beams suggests that these waves are generated through a local resonant process with the streaming electrons. The resonant energy can be estimated through the condition of cyclotron resonance with electrons, $\omega-k_{\|} v_{\|}=N \Omega_{e}$, where $\omega$ is the wave frequency, $k_{\|}$and $v_{\|}$are the parallel components of the wave vector and the electron velocity, $\Omega_{e}$ is the electron cyclotron frequency, and $N$ is an integer $(0, \pm 1, \pm 2, \ldots)$.

We have studied the properties of these waves by analyzing the data for 08:00-09:00 UT of 17 March 2001, as an example. Figure 6 shows the variations of magnetic and electric fields for the period detected in a channel with central frequency of $140.3 \mathrm{~Hz}$, selected as an example of channels where the waves are clearly seen. In the period we selected 6 sub-intervals (labelled with 1 to 6 between pairs of vertical lines in the panels) for detailed study. In panel 3 we show the energy flux of electrons in the channel of $57.69 \mathrm{eV}$, plotted in three pitch angle ranges corresponding to those in panels 4 to 6 of Fig. 3. It is obvious that the wave enhancements occurred in a period of anisotropic electrons, with more electrons with pitch angle $<30^{\circ}$ (the blue curve) or $>150^{\circ}$ (the red curve) than those with $\sim 90^{\circ}$ pitch angle (the black curve). We notice that there is little anisotropy for the wave burst number 4, but from the spectra in Fig. 3, we see streaming electrons at lower energy $(30-40 \mathrm{eV})$ during this sub-interval (see the last panel in Fig. 3, between 08:40 and 08:50 UT). We also notice that not all intervals with strong anisotropy have wave enhancements, for example, the intervals between wave bursts 1 and 2 , and between bursts 2 and 3 . This may indicate that only the instabilities that satisfy the condition of cyclotron resonance will grow by interacting with streaming electrons while others will not grow large enough to be observed even though there are electrons streaming in the plasma.

The spectra of average amplitude density for the six intervals are displayed in the lower part of the figure, which show that the peak intensity of the waves lies at frequencies between 140 and $230 \mathrm{~Hz}$. For the waves at these peak frequencies, for each interval, we estimate the wave phase velocity by calculating the ratio of average $E$ and $B$ over the interval. Then the energy of resonant electrons is estimated for the lowest possible energy according to the resonant condition above. The index of refraction is also estimated with $c B / E$, where $c$ is the speed of light, and the values are compared with the index of refraction for the whistler mode waves, 
Table 1. Results of wave analyses for the six intervals in Fig. 6.

\begin{tabular}{llllllllll}
\hline & $\begin{array}{l}\text { Time Interval } \\
(\mathrm{UT})\end{array}$ & $\begin{array}{l}\mathrm{DC}<\mathrm{B}> \\
(\mathrm{nT})\end{array}$ & $\begin{array}{l}<\text { Density }> \\
\left(/ \mathrm{cm}^{3}\right)\end{array}$ & $\begin{array}{l}\text { f_peak } \\
(\mathrm{Hz})\end{array}$ & $\begin{array}{l}\text { V_phase } \\
(\mathrm{c})\end{array}$ & $\begin{array}{l}\mathrm{e}^{-} \text {Energy } \\
(\mathrm{eV})\end{array}$ & $\mathrm{cB} / \mathrm{E}$ & $\mathrm{n}\left(\theta=40^{\circ}\right)$ & $\mathrm{n}\left(\theta=0^{\circ}\right)$ \\
\hline 1 & $08: 11: 23-08: 12: 36$ & 19.08 & 0.29 & 176.78 & 0.023 & 134.8 & 43.57 & 23.62 & 19.05 \\
2 & $08: 20: 24-08: 21: 35$ & 19.42 & 0.32 & 176.78 & 0.019 & 88.6 & 53.74 & 24.79 & 20.03 \\
3 & $08: 28: 12-08: 30: 00$ & 18.66 & 0.53 & 222.73 & 0.013 & 41.8 & 78.23 & 32.89 & 25.31 \\
4 & $08: 41: 59-08: 43: 47$ & 17.11 & 0.26 & 140.31 & 0.012 & 39.4 & 80.62 & 25.68 & 21.01 \\
5 & $08: 55: 12-08: 56: 23$ & 19.46 & 0.46 & 140.31 & 0.014 & 47.1 & 73.68 & 30.92 & 25.59 \\
6 & $08: 57: 36-08: 58: 47$ & 20.01 & 0.53 & 176.78 & 0.015 & 59.7 & 65.45 & 30.93 & 25.09 \\
\hline
\end{tabular}

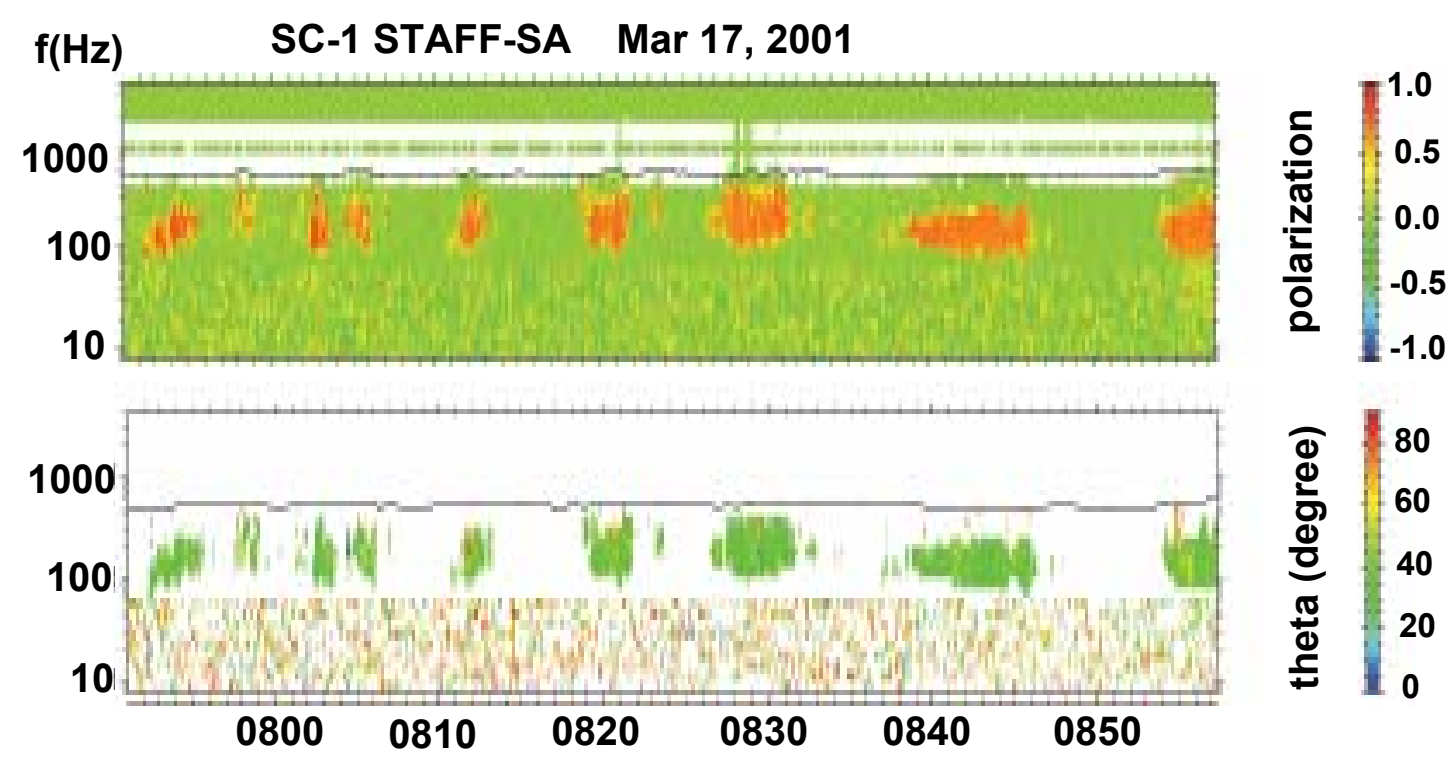

Fig. 7. Results from computer program PRASSADCO showing the polarization (upper panel) and the wave angle (lower panel) of the waves for the interval on 17 March 2001, 07:51-08:57 UT.

calculated as $n=\left[\omega_{p}^{2} / \omega\left(\Omega_{e} \cos \theta-\omega\right)\right]^{1 / 2}$, where $\omega_{p}$ is the electron plasma frequency, and $\theta$ is the wave angle. The results of these calculations are listed in Table 1.

The results show that the phase velocity of the waves is about 0.01-0.02 times the speed of light. The energy of the resonant electrons is estimated as $\sim 40 \mathrm{eV}$ to $140 \mathrm{eV}$. This energy range agrees well with the energy range of electron beams observed in the same period (Fig. 3). The calculated $c B / E$ from data is somewhat larger, by a factor of 2-3 than that calculated from the whistler dispersion relation, but is still comparable. There are some uncertainties in the estimation. The electric field wave data used are the sum of two components only, while the magnetic wave data are the sum of three components. This may cause overestimates of $c B / E$. Also, the use of average values may cause the discrepancies between the results from data and from theoretical calculations, since the wave bursts might be much shorter than the averaging intervals. We also notice that larger wave angles increase the value of the index of refraction, as can be seen from the last two columns of Table 1. The results seem to suggest that these waves propagate in slightly oblique direction, with wave angles ranging from 30 to $50 \mathrm{deg}$. Considering these uncertainties, we identify these CBL waves as a whistler mode excited through resonant interaction with the electron beams of several tens of $\mathrm{eV}$ to a few hundred $\mathrm{eV}$.

The Cluster's STAFF team has developed a computer program PRASSADCO to analyze multi-component measurements of electromagnetic waves, which can be used to estimate the wave vector direction, the degree and sense of the wave polarization and other propagation parameters (see Cornileau-Wehrlin et al., 2003; Santolik et al., 2001; Maksimovic et al., 2001, and references therein). We have applied the program to the above period to see the wave angle and the polarization of these CBL whistler waves. The results are 
shown in Fig. 7. The wave angle (the lower panel) is plotted with red color representing $\theta=90^{\circ}$ and blue for $\theta=0^{\circ}$. It is clear that the majority of these waves are propagating with wave angles of about $30^{\circ}-40^{\circ}$. The upper panel shows the polarization of the waves, with red (value of 1.0) representing right hand polarized waves, and blue (value of -1 ) for left handed waves. The results show that the CBL waves are right handed polarized, a typical whistler mode signature.

Tsurutani et al. (1998a, 2001) reported observations of polar cap boundary layer (PCBL) waves, which are narrow band electromagnetic emissions at frequencies just below $f_{c e}$. They are identified as the whistler mode generated probably by a cyclotron resonant interaction with electron beams, which are estimated to have energy of $\sim 100 \mathrm{eV}$ to $1 \mathrm{keV}$. In some case studies of the PCBL wave events, the phase velocity of the waves is about $0.01 c$, similar to those in our cases. These similarities indicate that these waves may be of the same nature.

Narrow band electrostatic emissions at slightly above $f_{c e}$ have been observed, mostly seen in the closed boundary layer. These waves have often been observed in previous studies (e.g. in Gurnett and Frank, 1978; Menietti et al., 2002a). Gurnett and Frank (1978) identified the waves as electron cyclotron waves occurring in the cusp and extending to the magnetosphere and polar cap region. Menietti et al. (2002a) suggested the waves were generated by low energy electron beams $(<1 \mathrm{keV})$. Similar waves are also observed in low altitude (Pottelette et al., 1990). The generation of these waves was attributed to the Bernstein mode or electron cyclotron instabilities, which may excite electrostatic waves above $f_{c e}$. Young et al. (1973) suggested that warm electron distribution with $\mathrm{df} / \mathrm{dv}_{\perp}>0$ can excite such waves with $f_{c e}<f<1.5 f_{c e}$. In the two events in our study, the narrow band electrostatic wave bursts are only observed in a few short intervals, but the electrostatic nature and the frequencies of the waves being near and above $f_{c e}$ clearly indicate that they are the same type of waves mentioned above.

"Lion roars" are also observed in the high latitude magnetosheath near the cusp. As in the case in low latitude magnetosheath (Tsurutani et al., 1982), the lion roars in high latitude magnetosheath also often occur in the dips of mirror mode waves, as well as in other regions of magnetosheath which are not magnetic dips (Maksimovic et al., 2001; Zhang et al., 1998). We notice that although the plasma conditions in the cusp and in the magnetosheath are similar, lion roars are not observed in the cusp.

Although we have presented here only two events of polar cusp crossings, we believe that the distinct waves occurring in various regions in the cusp vicinity as described in this study are typical and are commonly seen along Cluster's trajectories of high altitude cusp crossings. Thus observations of these waves may also serve as indicators of various regions in the vicinity of the cusp.

Acknowledgements. The research at UC Berkeley is funded under a NASA grant to the University of California NNG04GF23G and NAG5-12004. N. Lin would like to thank P. Canu for providing Whisper data for this study and for helpful discussion. We also thank the referees for their constructive comments and suggestions.

Topical Editor I. A. Daglis thanks M. L. Kaiser and another referee for their help in evaluating this paper.

\section{References}

Balogh A., Dunlop, M. W., Cowley, S. W. H., et al.: The Cluster Magnetic Field Investigation, Space Sci. Rev., 79, 65-92, 1997.

Cattell, C. A., Dombeck, J., Wygant, J. R., Hudson, M. K., Mozer, F. S., Temerin, M. A., Peterson, W. K., Kletzing, C. A., Russell, C. T., Pfaff, R. F.: Comparisons of Polar satellite observations of solitary wave velocities in the plasma sheet boundary and the high altitude cusp to those in the auroral zone, Geophys. Res. Lett., 26, 425-428, 1999.

Cornilleau-Wehrlin, N., Chauveau, P., Louis, S., et al.: The Cluster Spatio-temporal Analysis of Field Fluctuations (STAFF) experiment, Space Sci. Rev., 79, 107-136, 1997.

Cornilleau-Wehrlin N., Chanteur, G., Perraut, S., et al.: First Results Obtained by the Cluster STAFF Experiment, Ann. Geophys., 21, 437-456, 2003.

Crooker, N. U.: The Magnetospheric Boundary Layers: A Geometrically Explicit Model, J. Geophys. Res., 82, 3629-3633, 1977.

Franz, J. R., Kintner, P. M., and Pickett, J. S.: POLAR observations of coherent electric field structures, Geophys. Res. Lett., 25(8), 1277-1280, 1998.

Grison, B., Sahraoui, F., Lavraud, B., Chust, T., CornilleauWehrlin, N., Rème, H., Balogh, A., and Andre, M.: Wave particle interactions in the high-altitude polar cusp: a Cluster case study, Ann. Geophys., 3699-3713, 2005

Gurnett, D. A. and Frank, L. A.: Plasma Waves in the Polar Cusp: Observations From Hawkeye 1, J. Geophys. Res., 83, 14471462, 1978.

Gustafsson, G., André, M., Carozzi, T., et al.: First results of electric field and density observations by Cluster EFW based on initial months of operation, Ann. Geophys., 19, 1219-1240, 2001.

Johnstone, A. D., Alsop, C., Burge, S., et al.: PEACE: A Plasma Electron and Current Experiment, Space Sci. Rev., 79, 351-398, 1997.

Khotyaintsev, K., Vaivads, A., Ogawa, Y., Popielawska, B., André, M., Buchert, S., Décréau, P., Lavraud, B., and Rème, H.: Cluster observations of high-frequency waves in the exterior cusp, Ann. Geophys., 22, 2403-2411, 2004.

Lakhina, G. S., Tsurutani, B. T., Kojima, H., Matsumoto, H.: "Broadband" plasma waves in the boundary layers, J. Geophys. Res., 105, 27 791-27 831, 2000.

Lakhina, G. S., Tsurutani, B. T., and Pickett, J. S.: Association of Alfven waves and proton cyclotron waves with electrostatic bipolar pulses: magnetic hole events observed by Polar, Nonlin. Proc. Geophys., 11, 205-213, 2004.

Lavraud, B., Phan, T. D., Dunlop, M. W., et al.: The exterior cusp and its boundary with the magnetosheath: Cluster multi-event analysis, Ann. Geophys., 22, 3039-3054, 2004. 
Maksimovic, M., Harvey, C. C., Santolik, O., et al.: Polarization and Propagation of Lion Roars in the Dusk Side Magnetosheath, Ann. Geophys., 19, 1429-1438, 2001.

Matsumoto, H., Kojima, H., Miyatake, T., Omura, Y., Okada, M., Nagano, I., and Tsutsui, M.: Electrostatic solitary waves (ESW) in the magnetotail: BEN wave forms observed by GEOTAIL, Geophys. Res. Lett., 21, 2915-2918, 1994.

Menietti, J. D., Santolik, O., Scudder, J. D., Pickett, J. S., and Gurnett, D. A.: Electrostatic electron cyclotron waves generated by low-energy electron beams, J. Geophys. Res., 107(A10), 1285, doi:10.1029,/2001JA0092223, 2002a.

Menietti, J. D., Santolik, O., Scudder, J. D., Pickett, J. S., and Gurnett, D. A.: Correction to "Electrostatic electron cyclotron waves generated by low-energy electron beams" by Menietti, J. D. et al., J. Geophys. Res., 107(A12), 1462, doi:10.1029/2002JA009770, $2002 b$.

Onsager, T. G., Scudder, J. D., Lockwood, M., and Russell, C. T.: Reconnection at the high-latitude magnetopause during northward interplanetary magnetic field conditions, J. Geophys. Res. Vol. 106, No. A11, 25 467-25 488, 2001.

Pickett, J. S., Franz, J. R., Scudder, J. D., Menietti, J. D., Gurnett, D. A., Hospodarsky, G. B., Braunger, R. M., Kintner, P. M., and Kurth, W. S.: Plasma Waves Observed in the Cusp Turbulent Boundary Layer: An Analysis of High Time Resolution Wave and Particle Measurements from the Polar Spacecraft, J. Geophys. Res., 106, 19081-19 099, 2001.

Pickett, J. S., Menietti, J. D., Hospodarsky, G. B., Gurnett, D. A., and Stasiewicz, K.: Analysis of the turbulence observed in the outer cusp turbulent boundary layer, Adv. Space Res., 30, 28092814, 2002.

Potemra, T. A.: Sources of large-scale Birkeland currents, in Physical Signatures of Magenetospheric Boundary Layer Processes, NATO ASI Series, edited by: Holtet, J. A. and Egeland, A., Kluwer Academic Publishers, 3-27, 1994.

Pottelette, R., Malingre, M., Dubouloz, N., Aparicio, B., Lundin, R., Holmgren, G., and Marklund, G.: High-frequency Waves in the Cusp/Cleft Regions, J. Geophys. Res., 95, 5957-5971, 1990.

Réme, H., Bosqued, J. M., Sauvaud, J. A., et al.: The Cluster Ion Spectrometry (CIS) Experiment, Space Sci. Rev., 79, 303-350, 1997.

Réme, H., Aoustin, C., Bosqued, J. M., et al.: First multispacecraft measurements in and near the Earth's magnetosphere with the identical Cluster ion spectrometry (CIS) experiment, Ann. Geophys., 19, 1303-1354, 2001.

Santolik, O., Lefeuvre, F., Parrot M., and Rauch, J. L.: Complete wave-vector directions of electromagnetic emissions: Application to Interball-2 measurements in the nightside auroral zone, J. Geophys. Res., 106, 13 191-13 201, 2001.

Scarf, F. L., Fredricks, R. W., Green, I. M., and Russell, C. T.: Plasma Waves in the Dayside Polar Cusp 1. Magnetospheric Observations, J. Geophys. Res., 77, 2274-2293, 1972.
Scarf, F. L., Fredricks, R. W., and Neugebauer, M.: Plasma Waves in the Dayside Polar Cusp 2. Magnetopause and Polar Magnetosheath, J. Geophys. Res., 79, 511-520, 1974.

Stasiewicz, K., Seyler, C. E., Mozer, F. S., Gustafsson, G., Pickett, J., and Popielawska, B.: Magnetic bubbles and kinetic Alfvén waves in the high-latitude magnetopause boundary, J. Geophys. Res., 106(A12), 29 503-29514, doi:10.1029/2001JA900055, 2001.

Tsurutani, B. T., Smith, E. J., Anderson, R. R., Ogilvie, K. W., Scudder, J. D., Baker, D. N., and Bame, S. J.: Lion Roars and Nonoscillatory Drift Mirror Waves in the Magnetosheath, J. Geophys. Res., 87, 6060-6072, 1982.

Tsurutani, B. T., Arballo, J. K., Lakhina, G. S., Ho, C. M., Buti, B., Pickett, J. S., and Gurnett, D. A.: Plasma waves in the dayside polar cap boundary layer: Bipolar and monopolar electric pulses and whistler mode waves, Geophys. Res. Lett., 25, 4117-4120, 1998a.

Tsurutani, B. T., Lakhina, G. S., Ho, C. M., et al.: Broadband plasma waves observed in the polar cap boundary layer : Polar, J. Geophys. Res., 103, 17 351-17 366, 1998b.

Tsurutani, B. T., Arballo, J. K., Galvan, C., Zhang, L. D., Zhou, X. Y., Lakhina, G. S., Hada, T., Pickett, J. S., and Gurnett, D. A: Polar cap boundary layer waves: An auroral zone phenomenon, J. Geophys. Res., 106, 19 035-19055, 2001.

Tsurutani, B. T., Dasgupta, B., Arballo, J. K., Lakhina, G. S., and Pickett, J. S.: Magnetic field turbulence, electron heating, magnetic holes, proton cyclotron waves, and the onsets of bipolar pulse (electron hole) events: a possible unifying scenario, Nonlin. Proc. Geophys., 21, 27-35, 2003.

Vontrat-Reberac, A., Bosqued, J. M., Taylor, M. G., Lavraud, B., Fontaine, D., Dunlop, M. W., Laakso, H., CornilleauWerhlin, N., Canu, P., and Fazarkerley, A.: Cluster observations of the high-altitude cusp for northward interplanetary magnetic field: a case study, J. Geophys. Res., 108 (A9), 1346, doi:10.1029/2002JA009717, 2003.

Yearby, K. H., Alleyne, H. S. C. K., Cornilleau-Wehrlin, N., Santolik, O., Balikhin, M. A., Walker, S. N., Fazakerley, A., and Lahiff, A.: Observations of lion roars in the magnetosheath by the STAFF/DWP experiment on the Double Star TC-1 spacecraft, Ann. Geophys., 23, 2861-2866, 2005.

Young, T. S. T., Callen, J. D., and McCune, J. E.: High frequency electrostatic waves in the magnetosphere, J. Geophys. Res., 78, 1082-1099, 1973.

Zhang, Y., Matsumoto, H., and Kojima, H.: Lion roars in the magnetosheath: the Geotail observations, J. Geophys. Res., 103, 4615-4626, 1998.

Zong, Q.-G., Fritz, T. A., Spence, H., Korth, A., and Daly. P.: Energetic Electrons as a Field Line Topology Tracer in the Cusp Region: Cluster Rapid Observation, Survey Geophysics, 26, 126, 2005. 